\title{
Study of Differential Equations on Warped Product Semi- Invariant Submanifolds of the Generalized Sasakian Space Forms
}

\author{
Ibrahim Al-Dayel \\ Department of Mathematics and Statistics, College of Science, Imam Mohammad Ibn Saud Islamic University (IMSIU), \\ Riyadh 11566, Saudi Arabia \\ Correspondence should be addressed to Ibrahim Al-Dayel; iaaldayel@imamu.edu.sa
}

Received 28 May 2021; Accepted 19 July 2021; Published 2 August 2021

Academic Editor: Meraj Ali Khan

Copyright (C) 2021 Ibrahim Al-Dayel. This is an open access article distributed under the Creative Commons Attribution License, which permits unrestricted use, distribution, and reproduction in any medium, provided the original work is properly cited.

\begin{abstract}
The purpose of the present paper is to study the applications of Ricci curvature inequalities of warped product semi-invariant product submanifolds in terms of some differential equations. More precisely, by analyzing Bochner's formula on these inequalities, we demonstrate that, under certain conditions, the base of these submanifolds is isometric to Euclidean space. We also look at the effects of certain differential equations on warped product semi-invariant product submanifolds and show that the base is isometric to a special type of warped product under some geometric conditions.
\end{abstract}

\section{Introduction}

Bishop and O’Neill [1] evaluated the geometry of manifolds having negative curvature and noticed that Riemannian product manifolds do have nonnegative curvature. As a result, they came up with the recommendation of warped product manifolds, which are described as follows.

Consider two Riemannian manifolds $\left(L_{1}, g_{1}\right)$ and $\left(L_{2}\right.$, $g_{2}$ ) with corresponding Riemannian metrics $g_{1}$ and $g_{2}$ and $\psi: L_{1} \longrightarrow R$ be a positive differentiable function. If $x$ and $y$ are projection maps such that $x: L_{1} \times L_{2} \longrightarrow L_{1}$ and $y: L_{1}$ $\times L_{2} \longrightarrow L_{2}$, which are defined as $x(m, n)=m$ and $y(m, n)$ $=n \forall(m, n) \in L_{1} \times L_{2}$, then, $\bar{L}=L_{1} \times L_{2}$ is called warped product manifold if the Riemannian structure on $L$ satisfies

$$
g(\bar{E}, \bar{F})=g_{1}\left(x_{*} \bar{E}, x_{*} \bar{F}\right)+(\psi \circ x)^{2} g_{2}\left(y_{*} \bar{E}, y_{*} \bar{F}\right)
$$

for all $\bar{E}, \bar{F} \in T \bar{L}$. The function $\psi$ represents the warping function of $L_{T}^{n_{1}} \times L_{2}$. The Riemannian product manifold is a special case of warped product manifold in which the warping function $\psi=1$. The study of Bishop and O'Neill [1] revealed that these types of manifolds have a wide range of applications in physics and theory of relativity. It is well known that the warping function is the solution of some partial differential equations; for example, the Einstein field equation can be solved by the approach of warped product [2]. The warped product is also applicable in the study of space time near black holes [3].

On the other hand, the analysis of differential equation on Riemannian manifolds yields some important geometric and isometric intrinsic properties. It is well known that categorization of differential equation has a major influence on the global analysis of Riemannian manifolds. Tanno [4] explored various aspects of differential equations on Riemannian manifolds in 1978. The approach of differential equations was used by the authors $[5,6]$ to describe the Euclidean sphere. These calculations demonstrated that a nonconstant function $\lambda$ on a complete Riemannian manifold $\left(U^{n}, g\right)$ satisfies the differential equation as follows:

$$
\nabla^{2} \lambda+k g=0
$$

if and only if $\left(U^{n}, g\right)$ is congruent to Euclidean space $R^{n}$, where $k$ is constant.

Furthermore, under some geometric conditions, GarciaRio et al. [6] proved that the Riemannian manifold is isometric to the warped product $U \times{ }_{f} R$, where $U$ is a complete 
Riemannian manifold, $R$ is the Euclidean line, and $f$ is the warping function. Moreover, warping function $f$ is the solution of the following differential equation:

$$
\frac{d^{2} f}{d t^{2}}+\mu_{1} f=0
$$

if and only if there exists a nonconstant function $\phi: U^{n}$ $\longrightarrow R$ with an eigenvalue $\lambda_{1}<0$, which satisfies the following differential equation:

$$
\Delta \phi+\mu_{1} \phi=0
$$

The categorization of differential equations on Riemannian manifolds turns into an attractive research subject that has been explored by various researchers, for example, [7-11].

Al-Dayel et al. [7] recently investigated the effect of the differential equation (3) on the Riemannian manifold $\left(L^{n}, g\right)$ using the concircular vector field, showing that the Riemannian manifold $\left(L^{n}, g\right)$ is isometric to the Euclidean manifold $R^{n}$. By using the gradient conformal vector field, Chen et al. [12] discovered that the Riemannian manifold $\left(L^{n}, g\right)$ is isometric to the Euclidean space $R^{n}$. However, it has been shown in [13] that the complete totally real submanifold in $\mathrm{CP}^{n}$ (complex projective space) with bounded Ricci curvature satisfying (4) is isometric to a special form of hyperbolic space.

Latterly, Ali et al. [8] characterized warped product submanifolds in Sasakian space form by the approach of differential equation. The purpose of this paper is to study the impact of differential equation on warped product semi-invariant product submanifolds in the framework of generalized Sasakian space form.

\section{Preliminaries}

A $(2 k+1)$-dimensional $C^{\infty}$-manifold $\bar{L}$ is said to have an almost contact structure if there exists on $\bar{L}$ a tensor field $\phi$ of the type $(1,1)$, a vector field $\chi$, and a 1 -form $\eta$ satisfying

$$
\begin{aligned}
\phi^{2} & =-I+\eta \otimes \chi, \\
\phi \chi & =0, \\
\eta \circ \phi & =0, \\
\eta(\chi) & =1 .
\end{aligned}
$$

On an almost contact metric manifold $\bar{L}$, there is always a Riemannian metric $g$ that meets the following requirements:

$$
\begin{aligned}
\eta(E) & =g(E, \chi), \\
g(\phi E, \phi F) & =g(E, F)-\eta(E) \eta(F),
\end{aligned}
$$

for all $E, F \in T \bar{L}$.
An almost contact metric manifold is said to be nearly Sasakian manifold, if

$$
\left(\bar{\nabla}_{E} \phi\right) F+\left(\bar{\nabla}_{F} \phi\right) E=-2 g(E, F) \chi+\eta(F) E+\eta(E) F
$$

for all $E, F \in T \bar{L}$.

In [14], Alegre et al. gave the concept of generalized Sasakian space form as that an almost contact metric manifold $(\bar{L}, f, \chi, \eta, g)$ whose curvature tensor $\bar{R}$ satisfies

$$
\begin{aligned}
\bar{R}(E, F, G, W)= & \phi_{1}[g(F, G) g(E, W)-g(E, G) g(F, W)] \\
& -\phi_{2}[g(\phi E, G) g(\phi F, W)-g(\phi E, W) g(\phi F, G) \\
& +2 g(\phi E, F) g(\phi G, W)]-\phi_{3}[\eta(G)\{\eta(F) g(E, W) \\
& -\eta(E) g(G, W)\}+\eta(W)\{\eta(E) g(F, G)-\eta(F) g(E, G)\}]
\end{aligned}
$$

for any vector fields $E, F, G, W$ and certain differentiable functions $\phi_{1}, \phi_{2}, \phi_{3}$ on $\bar{L}$. A generalized Sasakian space form with functions $\phi_{1}, \phi_{2}, \phi_{3}$ is denoted by $\bar{L}\left(\phi_{1}, \phi_{2}, \phi_{3}\right)$. If $\phi_{1}=$ $(c+3) / 4, \phi_{2}=\phi_{3}=(c-1) / 4$, then $\bar{M}\left(\phi_{1}, \phi_{2}, \phi_{3}\right)$ is a Sasakian space form $\bar{M}(c)$ [14]. If $\phi_{=}(c-3) / 4, \phi_{2}=\phi_{3}=(c+1) / 4$, then $\bar{M}\left(\phi_{1}, \phi_{2}, \phi_{3}\right)$ is a Kenmotsu space form $\bar{M}(c)$ [14], and if $\phi_{1}=\phi_{2}=\phi_{3}=c / 4$, then $\bar{M}\left(\phi_{1}, \phi_{2}, \phi_{3}\right)$ is a cosymplectic space form $\bar{M}(c)$ [14].

A submanifold $L$ of an almost contact metric manifold $\bar{L}$ is called semi-invariant submanifolds (contact CR-submanifolds) if there exist two orthogonal complementary distributions $D$ and $D^{\perp}$ satisfying the following conditions:

(i) $T L=D \oplus D^{\perp} \oplus\langle\xi\rangle$, where $\langle\xi\rangle$ is the distribution spanned by the structure vector field $\xi$

(ii) $D$ is invariant distribution, i.e., $\phi D \subset T L$

(iii) $D^{\perp}$ is anti-invariant, i.e., $\phi D^{\perp} \subseteq T^{\perp} L$

Recently, we [15] studied warped product semi-invariant product submanifolds of the type $L^{n}=L_{T}^{n_{1}} \times{ }_{f} L_{\perp}^{n_{2}}$ isometrically immersed in the generalized Sasakian space form admitting a nearly Sasakian structure, where $L_{T}^{n_{1}}$ is an invariant submanifold of dimension $n_{1}$ and $L_{\perp}^{n_{2}}$ is a totally real submanifold of dimension $n_{2}$. More precisely, the computed Ricci curvature inequalities for these submanifolds are as follows:

Theorem 1. Let $L^{n}=L_{T}^{n_{1}} \times{ }_{f} L_{\perp}^{n_{2}}$ be a warped product semiinvariant submanifold isometrically immersed in a generalized Sasakian space form $\bar{L}^{m}\left(\phi_{1}, \phi_{2}, \phi_{3}\right)$ with nearly Sasakian structure. Then, for each orthogonal unit vector field $\xi \in T_{x} M$ orthogonal to $\chi$, either tangent to $N_{T}^{n_{1}}$ or $N_{\perp}^{n_{2}}$, the Ricci curvature satisfies the following inequalities:

(i) If $\xi \in T L_{T}^{n_{1}}$, then 


$$
\begin{aligned}
\operatorname{Ric}(\xi) \leq & \frac{1}{4} n^{2}\|H\|^{2}-n_{2} \Delta \ln f+n_{2}\|\nabla \ln f\|^{2}+\frac{3 \phi_{2}}{2} \\
& +\left(n+n_{1} n_{2}-1\right) \phi_{1}-\left(n_{2}+1\right) \phi_{3}
\end{aligned}
$$

(ii) If $\xi \in T L_{\perp}^{n_{2}}$, then

$$
\begin{aligned}
\operatorname{Ric}(\xi) \leq & \frac{1}{4} n^{2}\|H\|^{2}-n_{2} \Delta \ln f+n_{2}\|\nabla \ln f\|^{2} \\
& +\left(n+n_{1} n_{2}-1\right) \phi_{1}-\left(n_{2}+1\right) \phi_{3}
\end{aligned}
$$

The equality cases can be seen in [15].

Let $f$ be a real-valued differential function on a Riemannian manifold $L^{n}$, then the Bochner formula [16] is stated as

$$
\frac{1}{2} \Delta|\nabla f|^{2}=R^{L}(\nabla f, \nabla f)+|H(f)|^{2}+g(\nabla \Delta f, \nabla f)
$$

where $R^{L}$ denotes Ricci tensor and $H(f)$ is the Hessian of the function $f$.

\section{Main Results}

In this section, we obtain some characterization by the application of Bochner's formula.

Theorem 2. Let $L^{n}=L_{T}^{n_{1}} \times{ }_{f} L_{\perp}^{n_{2}}$ be a $n$-dimensional warped product semi-invariant product submanifold in a generalized Sasakian space form $\bar{L}^{m}(c)$, where $L_{T}^{n_{1}}$ is a $n$-dimensional invariant submanifold and $L_{\perp}^{n_{2}}$ is an anti-invariant submanifold. Such that Ricci curvature $R^{L}(\xi) \geq b, b>0$. If $\xi \in T L_{T}^{n_{1}}$ and satisfying the following equality:

$\left(\lambda_{1}+n_{2}\right) b=\lambda_{1}\left[\frac{n_{2}}{n}+\frac{n^{2}}{4}\|H\|^{2}-\left(n+n_{1} n_{2}-1\right) \phi_{1}-\frac{3 \phi_{2}}{2}+\left(n_{2}+1\right) \phi_{3}\right]$,

then, the base submanifold $L_{T}^{n_{1}}$ is isometric to $R^{n_{1}}$ (Euclidean space).

Proof. Since $\xi \in T N_{T}^{n_{1}}$, by equation (9)

$$
\begin{aligned}
R^{L}(\xi)+n_{2} \Delta \ln f \leq & \frac{1}{4} n^{2}\|H\|^{2}+n_{2}\|\nabla \ln f\|^{2}+\left(n+n_{1} n_{2}-1\right) \phi_{1} \\
& +\frac{3 \phi_{2}}{2}-\left(n_{2}+1\right) \phi_{3} .
\end{aligned}
$$

By the assumption that $R_{L}(\xi) \geq b$, we have

$$
\begin{aligned}
b+n_{2} \Delta \ln f \leq & \frac{1}{4} n^{2}\|H\|^{2}+n_{2}\|\nabla \ln f\|^{2}+\left(n+n_{1} n_{2}-1\right) \phi_{1} \\
& +\frac{3 \phi_{2}}{2}-\left(n_{2}+1\right) \phi_{3} .
\end{aligned}
$$

Since the Ricci curvature $R^{L}(\xi)$ is bounded below by $b \geq 0$, then by virtue of theorem of Myers [17], the base manifold $L_{T}^{n_{1}}$ is compact. On integrating (9) and using Green's theorem, we have

$$
\begin{aligned}
\operatorname{Vol}\left(L_{T}^{n_{1}}\right) b \leq & \frac{n^{2}}{4} \int_{L_{T}^{n_{1}} \times\{q\}}\|H\|^{2} d V+n_{2} \int_{L_{T}^{n_{1}} \times\{q\}}\|\nabla \ln f\|^{2} d V \\
& +\int_{L_{T}^{n_{1}} \times\{q\}}\left[\left(n+n_{1} n_{2}-1\right) \phi_{1}+\frac{3 \phi_{2}}{2}-\left(n_{2}+1\right) \phi_{3}\right] d V,
\end{aligned}
$$

or

$$
\begin{aligned}
\int_{L_{T}^{n_{1}} \times\{q\}}\|\nabla \ln f\|^{2} d V \geq & \frac{b}{n_{2}} \operatorname{Vol}\left(L_{T}^{n_{1}}\right)-\frac{n^{2}}{4 n_{2}} \int_{L_{T}^{n_{1}} \times\{q\}}\|H\|^{2} d V-\frac{1}{n_{2}} \\
& \cdot \int_{L_{T}^{n_{1}} \times\{q\}}\left[\left(n+n_{1} n_{2}-1\right) \phi_{1}+\frac{3 \phi_{2}}{2}-\left(n_{2}+1\right) \phi_{3}\right] d V .
\end{aligned}
$$

Suppose $H(\ln f)$ denotes the Hessian of the warping function $\ln f$, then we have

$$
|H(\ln f)-n I|^{2}=|H(\ln f)|^{2}+n^{2}|I|^{2}-2 n g(I, H(\ln f)),
$$

after some calculations, the above formula turns to

$$
|H(\ln f)-t I|^{2}=2 t \Delta(\ln f)+t^{2}\left(n_{1}\right)+|H(\ln f)|^{2} .
$$

Putting $t=\lambda_{1} / n_{1}$ and integrating the last equation with respect to $d V$ (volume element), we get

$\int_{L_{T}^{n_{1}} \times\{q\}}\left|H(\ln f)-\frac{\lambda_{1}}{n_{1}} I\right|^{2} d V=\int_{L_{T}^{n_{1}} \times\{q\}}|H(\ln f)|^{2} d V+\int_{L_{T}^{n_{1}} \times\{q\}} \frac{\lambda_{1}^{2}}{n_{1}} d V$,

using (11), with the fact $\Delta \ln f=\lambda_{1} \ln f$, we have

$$
\int_{L_{T}^{n_{1}} \times\{q\}}|H(\ln f)|^{2} d V=-\lambda_{1} \int_{L_{T}^{n_{1}} \times\{q\}}|\nabla \ln f|^{2} d V-\int_{L_{T}^{n_{1}} \times\{q\}} R^{L}(\nabla \ln f, \nabla \ln f) .
$$

Merging (19) and (20), we derive

$$
\begin{aligned}
\int_{L_{T}^{n_{1}} \times\{q\}}\left|H(\ln f)-\frac{\lambda_{1}}{n_{1}} I\right|^{2} d V= & \int_{L_{T}^{n_{1}} \times\{q\}} \frac{\lambda_{1}^{2}}{n_{1}} d V-\lambda_{1} \int_{L_{T}^{n_{1}} \times\{q\}}|\nabla \ln f|^{2} d V \\
& -\int_{L_{T}^{n_{1}} \times\{q\}} R^{L}(\nabla f, \nabla f) d V .
\end{aligned}
$$

By the assumption $R^{L}(\nabla f, \nabla f) \geq b$, the above equation yields 


$$
\begin{aligned}
\int_{L_{T}^{n_{1}} \times\{q\}}\left|H(\ln f)-\frac{\lambda_{1}}{n_{1}} I\right|^{2} d V \leq & \int_{L_{T}^{n_{1}} \times\{q\}} \frac{\lambda_{1}^{2}}{n_{1}} d V-b \operatorname{Vol}\left(L_{T}^{n_{1}}\right) \\
& -\lambda_{1} \int_{L_{T}^{n_{1}} \times\{q\}}|\nabla \ln f|^{2} d V .
\end{aligned}
$$

Using (16), the last inequality leads to

$$
\begin{aligned}
\int_{L_{T}^{n_{1}} \times\{q\}}\left|H(\ln f)-\frac{\lambda_{1}}{n_{1}} I\right|^{2} d V \leq & \int_{L_{T}^{n_{1}} \times\{q\}} \frac{\lambda_{1}^{2}}{n_{1}} d V-\int_{L_{T}^{n_{1}} \times\{q\}}\left(\frac{\lambda_{1} b}{n_{2}}+b\right) d V-\frac{\lambda_{1}}{n_{2}} \\
& \cdot \int_{L_{T}^{n_{1}} \times\{q\}}\left[\left(n+n_{1} n_{2}-1\right) \phi_{1}+\frac{3 \phi_{2}}{2}-\left(n_{2}+1\right) \phi_{3}\right] d V \\
& -\frac{\lambda_{1} n^{2}}{4 n_{2}} \int_{L_{T}^{n_{1}} \times\{q\}}\|H\|^{2} d V .
\end{aligned}
$$

If (12) holds, then the above inequality produces

$$
\left|H(\ln f)-\frac{\lambda_{1}}{n_{1}} I\right|^{2}=0
$$

Therefore, we have $H(\ln f)(X, X)=\lambda_{1} / n_{1}$. Hence, by the application of the result of Tashiro [18], the fibre $L_{T}^{n_{1}}$ is isometric to $R^{n_{1}}$ (Euclidean space).

If we consider the unit vector field $\xi \in T L_{\perp}^{n_{2}}$, then we have the following results which can be proved by adopting similar steps in Theorem 2.

Theorem 3. Let $L^{n}=L_{T}^{n_{1}} \times{ }_{f} L_{\perp}^{n_{2}}$ be a $n$-dimensional warped product semi-invariant product submanifold in a generalized Sasakian space form $\bar{L}^{m}(c)$, where $L_{T}^{n_{1}}$ is a $n$-dimensional invariant submanifold and $L_{\perp}^{n_{2}}$ is an anti-invariant submanifold. Such that Ricci curvature $R^{L}(\xi) \geq b, b>0$. If $\xi \in T L_{T}^{n_{1}}$ and satisfying the following equality:

$$
\left(\lambda_{1}+n_{2}\right) b=\lambda_{1}\left[\frac{n_{2}}{n}+\frac{n^{2}}{4}\|H\|^{2}-\left(n+n_{1} n_{2}-1\right) \phi_{1}+\left(n_{2}+1\right) \phi_{3}\right],
$$

then, the base submanifold $L_{T}^{n_{1}}$ is isometric to $R^{n_{1}}$ (Euclidean space).

Now, we have the next result which is based on the study of Garcia-Rio et al. [6].

Theorem 4. Let $L^{n}=L_{T}^{n_{1}} \times{ }_{f} L_{\perp}^{n_{2}}$ be a warped product semiinvariant product submanifold in a generalized Sasakian space form admitting the nearly Sasakian structure $\bar{L}^{m}(c)$. Such that Ricci curvature $R^{L}(\xi)>b, b>0$. If $\xi \in T N_{T}^{n_{1}}$ and satisfying the following relation:

$$
\begin{aligned}
n^{2}\|H\|^{2}+\frac{4\left(n_{1} n_{2}\right)}{\lambda_{1}}|H(\ln f)|^{2} \\
\quad=\frac{4 n_{1} n_{2}}{\lambda_{1}}\left(b-\left(n+n_{1} n_{2}-1\right) \phi_{1}-\frac{3 \phi_{2}}{2}+\left(n_{2}+1\right) \phi_{3}\right),
\end{aligned}
$$

for $\lambda_{1}<0$, then $L_{T}^{n_{1}}$ is isometric to warped product of the type $R \times{ }_{\theta} U$ with the warping function $\theta$, which satisfies the differential equation $d \theta^{2} / d t^{2}+\lambda_{1} \theta=0$.

Proof. For the warping function $\ln f$, defining the following equation on $L_{T}^{n_{1}}$ :

$|b \ln f I+H(\ln f)|^{2}=b^{2}(\ln f)^{2}|I|^{2}+|H(\ln f)|^{2}+2 b(\ln f) g(I, H(\ln f))$.

But we know that $|I|^{2}=\operatorname{tr}\left(I I^{*}\right)=n_{1}$ and $g\left(H(\ln f), I^{*}\right)$ $=\operatorname{tr}\left(I^{*} H(\ln f)\right)=\operatorname{tr}(H(\ln f))$; using these facts, the above equation leads to

$$
|b \ln f I+H(\ln f)|^{2}=|H(\ln f)|^{2}+n_{1} b^{2}(\ln f)^{2}-2 b \ln f \Delta \ln f .
$$

Let $\ln f$ is an eigenfunction corresponding to the eigenvalue $\lambda_{1}$ satisfying $\Delta \ln f=\lambda_{1} \ln f$, we have

$$
|b \ln f I+H(\ln f)|^{2}=|H(\ln f)|^{2}+\left(n_{1} b^{2}-2 b \lambda_{1}\right)(\ln f)^{2} .
$$

Further, using $\Delta \ln f=\lambda_{1} \ln f$, it is easy to see that

$$
\nabla \frac{(\ln f)^{2}}{2}=\ln f \lambda_{1} \ln f-|\nabla \ln f|^{2}
$$

which on integrating provides

$$
\int_{L_{T}^{n_{1}} \times\{q\}}(\ln f)^{2} d V=\frac{1}{\lambda_{1}} \int_{L_{T}^{n_{1}} \times\{q\}}|\nabla \ln f|^{2}
$$

Thus, we have

$$
\begin{aligned}
\int_{L_{T}^{n_{1}} \times\{q\}}|H(\ln f)+b \ln f I|^{2} d V= & \int_{L_{T}^{n_{1}} \times\{q\}}|H(\ln f)|^{2} d V \\
& +\left(\frac{n_{1} b^{2}}{\lambda_{1}}-2 b\right) \\
& \cdot \int_{L_{T}^{n_{1}} \times\{q\}}|\nabla \ln f|^{2} d V .
\end{aligned}
$$


Choosing $b=\lambda_{1} / n_{1}$ in (32), we have

$$
\begin{aligned}
\int_{L_{T}^{n_{1}} \times\{q\}}\left|H(\ln f)+\frac{\lambda_{1}}{n_{1}} \ln f I\right|^{2} d V= & \int_{L_{T}^{n_{1}} \times\{q\}}|H(\ln f)|^{2} d V-\frac{\lambda_{1}}{n_{1}} \\
& \cdot \int_{L_{T}^{n_{1}} \times\{q\}}|\nabla \ln f|^{2} d V .
\end{aligned}
$$

Further, integrating (9) and applying Green's lemma, we find

$$
\begin{aligned}
\int_{L_{T}^{n_{1}} \times\{q\}} R^{L}(\xi) d V \leq & \frac{n^{2}}{4} \int_{L_{T}^{n_{1}} \times\{q\}}\|H\|^{2} d V+n_{2} \int_{L_{T}^{n_{1}} \times\{q\}}\|\nabla \ln f\|^{2} d V \\
& +\int_{L_{T}^{n_{1}} \times\{q\}}\left[\left(n+n_{1} n_{2}-1\right) \phi_{1}+\frac{3 \phi_{2}}{2}-\left(n_{2}+1\right) \phi_{3}\right] d V .
\end{aligned}
$$

From the above two expressions, we have

$$
\begin{aligned}
\frac{1}{n_{2}} \int_{L_{T}^{n_{1}} \times\{q\}} R^{L}(\xi) d V \leq & \frac{n^{2}}{4 n_{2}} \int_{L_{T}^{n_{1}} \times\{q\}}\|H\|^{2} d V+\frac{n_{1}}{\lambda_{1}} \int_{L_{T}^{n_{1}} \times\{q\}}|H(\ln f)|^{2} d V \\
& -\frac{n_{1}}{\lambda_{1}} \int_{L_{T}^{n_{1}} \times\{q\}}\left|H(\ln f)+\frac{\lambda_{1}}{n_{1}} \ln f I\right|^{2} d V+\int_{L_{T}^{n_{1}} \times\{q\}} \\
& \cdot\left[\left(n+n_{1} n_{2}-1\right) \phi_{1}+\frac{3 \phi_{2}}{2}-\left(n_{2}+1\right) \phi_{3}\right] d V .
\end{aligned}
$$

On using the assumption that $R^{L}(\xi) \geq b$, for $b>0$,

$$
\begin{aligned}
\int_{L_{T}^{n_{1}} \times\{q\}}\left|H(\ln f)+\frac{\lambda_{1}}{n_{1}} \ln f I\right|^{2} d V \leq & \frac{n^{2} \lambda_{1}}{4 n_{1} n_{2}} \int_{L_{T}^{n_{1}} \times\{q\}}\|H\|^{2} d V+\int_{L_{T}^{n_{1}} \times\{q\}}|H(\ln f)|^{2} d V \\
& -\frac{\lambda_{1}}{n_{1} n_{2}} \int_{L_{T}^{n_{1}} \times\{q\}} b d V+\frac{\lambda_{1}}{n_{1}} \int_{L_{T}^{n_{1}} \times\{q\}} \\
& \cdot\left[\left(n+n_{1} n_{2}-1\right) \phi_{1}+\frac{3 \phi_{2}}{2}-\left(n_{2}+1\right) \phi_{3}\right] d V,
\end{aligned}
$$

equivalently,

$$
\begin{aligned}
\int_{L_{T}^{n_{1}} \times\{q\}}\left|H(\ln f)+\frac{\lambda_{1}}{n_{1}} \ln f I\right|^{2} d V \leq \int_{L_{T}^{n_{1}} \times\{q\}} \\
\cdot\left\{\frac{\lambda_{1}}{n_{1}}\left(\frac{n^{2}}{4 n_{2}}\|H\|^{2}+\left(\left(n+n_{1} n_{2}-1\right) \phi_{1}+\frac{3 \phi_{2}}{2}-\left(n_{2}+1\right) \phi_{3}\right)\right)\right. \\
\left.+\frac{b}{n_{2}}+|H(\ln f)|^{2}\right\} d V .
\end{aligned}
$$

By assumption (26), we have

$$
\left|H(\ln f)+\frac{\lambda_{1}}{n_{1}} \ln f\right|^{2} \leq 0,
$$

which is not possible; therefore,

$$
H(\ln f)+\frac{\lambda_{1}}{n_{1}} \ln f I=0 .
$$

By taking trace of the above equation, we get

$$
\Delta \ln f+\lambda_{1} \ln f=0 .
$$

Now, applying the result proved in [6], together with the fact that $L^{t}=L_{T}^{n_{1}} \times{ }_{f} L_{\perp}^{n_{2}}$ is nontrivial, we deduced that $L_{T}^{n_{1}}$ is isometric to a warped product of the form $R \times{ }_{\theta} U$, where $U$ is complete Riemannian manifold. Moreover, the warping function $\theta$ is the solution of the differential equation $d \theta^{2} / d$ $t^{2}+\lambda_{1} \theta=0$. Hence, the proof is completed.

Similarly, we can prove the following theorems by taking the unit vector field $\xi$ tangent to $L_{\perp}^{n_{2}}$.

Theorem 5. Let $L^{n}=L_{T}^{n_{1}} \times{ }_{f} L_{\perp}^{n_{2}}$ be a warped product semiinvariant product submanifold in a generalized Sasakian space form admitting the nearly Sasakian structure $\bar{L}^{m}(c)$. Such that Ricci curvature $R^{L}(\xi)>b, b>0$. If $\xi \in T N_{\perp}^{n_{2}}$ and satisfying the following relation:

$$
\begin{aligned}
n^{2}\|H\|^{2}+ & \frac{4\left(n_{1} n_{2}\right)}{\lambda_{1}}|H(\ln f)|^{2}=\frac{4 n_{1} n_{2}}{\lambda_{1}} \\
& \cdot\left(b-\left(n+n_{1} n_{2}-1\right) \phi_{1}+\left(n_{2}+1\right) \phi_{3}\right),
\end{aligned}
$$

for $\lambda_{1}<0$, then, $L_{T}^{n_{1}}$ is isometric to warped product of the type $\mathbb{R} \times{ }_{\theta} U$ with the warping function $\theta$, which satisfies the differential equation $d \theta^{2} / d t^{2}+\lambda_{1} \theta=0$.

\section{Conclusions}

This paper studies the geometric behavior of ordinary differential equations on the warped product semi-invariant product submanifolds. More precisely, we obtain characterizing theorems for warped product semi-invariant product submanifolds of generalized Sasakian space forms via differential and integral theory on Riemannian manifolds. Therefore, the present article provides a wonderful correlation of the theory of differential equations with the warped product submanifolds.

\section{Data Availability}

No data were used to support this study.

\section{Conflicts of Interest}

The author declares that there is no conflict of interest regarding the publication of this paper. 


\section{References}

[1] R. L. O.'. N. B. Bishop, "Manifolds of negative curvature," Transactions of the American Mathematical Society, vol. 145, pp. 1-9, 1969.

[2] J. K. Beem, P. Ehrlich, and T. G. Powell, Warped Product Manifolds in Relativity Selected Studies, North-Holland, Amsterdam-New York, 1982.

[3] S. W. Hawkings and G. F. R. Ellis, The Large Scale Structure of Space-Time, Cambridge Univ. Press, Cambridge, 1973.

[4] S. Tanno, "Some differential equations on Riemannian manifolds," Journal of the Mathematical Society of Japan, vol. 30, no. 3, pp. 509-531, 1978.

[5] S. Deshmukh, "Characterizing spheres and Euclidean spaces by conformal vector fields," Annali di Matematica Pura ed Applicata (1923 -), vol. 196, no. 6, article 21352145, 2017.

[6] E. Garcia-Rio, D. N. Kupeli, and B. Unal, "On a differential equation characterizing Euclidean spheres," Journal of Differential Equations, vol. 194, no. 2, pp. 287-299, 2003.

[7] I. Al-Dayel, S. Deshmukh, and O. Belova, "A remarkable property of concircular vector fields on a Riemannian manifold," Mathematics, vol. 8, no. 4, p. 469, 2020.

[8] A. Ali, F. Mofarreh, W. A. Mior Othman, and D. S. Patra, "Applications of differential equations to characterize the base of warped product submanifolds of cosymplectic space forms," Journal of Inequalities and Applications, vol. 2020, no. 1, 2020.

[9] M. Jamali and M. H. Shahid, "Application of Bochner formula to generalized Sasakian space forms," Afrika Matematika, vol. 29, no. 7-8, pp. 1135-1139, 2018.

[10] A. Ali, F. Mofarreh, N. Alluhaibi, A. Ali, and I. Ahmad, "On differential equations characterizing Legendrian submanifolds of Sasakian space forms," Mathematics, vol. 8, no. 2, p. 150, 2020.

[11] P. Rudnicki and G. J. Woeginger, "The post correspondence problem over a unary alphabet," Applied Mathematics Letters, vol. 22, no. 5, pp. 723-727, 2009.

[12] B.-Y. Chen, S. Deshmukh, and A. A. Ishan, "On Jacobi-type vector fields on Riemannian manifolds," Mathematics, vol. 7, no. 12, p. 1139, 2019.

[13] Y. Matsuyama, "Complete totally real submanifolds of a complex projective space," Differential Geometry - Dynamical Systems, vol. 20, pp. 119-125, 2018.

[14] P. Alegre, D. E. Blair, and A. Carriazo, "Generalized Sasakian space forms," Israel Journal of Mathematics, vol. 141, pp. 1571-7183, 2004.

[15] A. Ibrahim and M. A. Khan, "Ricci curvature of contact CRwarped product submanifolds in generalized Sasakian space forms admitting nearly Sasakian structure," AIMS Mathematics, vol. 6, no. 3, pp. 2132-2155, 2021.

[16] M. Berger, "Les varietes riemanniennes (1/4)-pinces," Annali della Scuola Normale Superiore di Pisa - Classe di Scienze, vol. 14, no. 4, pp. 161-170, 1960.

[17] S. B. Myers, "Riemannian manifolds with positive mean curvature," Duke Mathematical Journal, vol. 8, no. 2, pp. 401-404, 1941.

[18] Y. Tashiro, "Complete Riemannian manifolds and some vector fields," Transactions of the American Mathematical Society, vol. 117, pp. 251-275, 1965. 